\title{
Propulsion Efficiency of a Dynamic Self-Assembled Helical Ribbon
}

\author{
Nebojsa Casic, ${ }^{1}$ Niurka Quintero, ${ }^{2}$ Renato Alvarez-Nodarse,${ }^{2}$ Franz G. Mertens, ${ }^{1}$ Levan Jibuti, ${ }^{1}$ \\ Walter Zimmermann, ${ }^{1}$ and Thomas M. Fischer ${ }^{1, *}$ \\ ${ }^{1}$ Institute of Physics, Universität Bayreuth, 95440 Bayreuth, Germany \\ ${ }^{2}$ Institute of Mathematics, University of Seville, E-41012 Seville, Spain
}

(Received 8 October 2012; published 15 April 2013)

\begin{abstract}
We study the dynamic self-assembly and propulsion of a ribbon formed from paramagnetic colloids in a dynamic magnetic field. The sedimented ribbon assembles due to time averaged dipolar interactions between the beads. The time dependence of the dipolar interactions together with hydrodynamic interactions cause a twisted ribbon conformation. Domain walls of high twist connect domains of nearly constant orientation and negligible twist and travel through the ribbon. The particular form of the domain walls can be controlled via the frequency and the eccentricity of the modulation. The flux of twist wallsa true ribbon property absent in slender bodies-provides the thrust onto the surrounding liquid that propels this biomimetic flagellum into the opposite direction. The propulsion efficiency increases with frequency and ceases abruptly at a critical frequency where the conformation changes discontinuously to a flat standing ribbon conformation.
\end{abstract}

DOI: 10.1103/PhysRevLett.110.168302

PACS numbers: 82.70.Dd, 87.15.hm

Nature dynamically self-assembles a rich variety of swimmers of different size and geometry [1,2]. Large swimmers move at a high Reynolds number. They generate eddies, thereby efficiently producing inertial thrust. Micron sized small swimmers lack these possibilities and must use nonreciprocal less efficient conformational dynamics [3-6]. Swimming strategies of low-Reynoldsnumber swimmers vary. "Squirmers" are stationary swimmers of large volume-to-surface ratio that maintain their shape. Propulsion is achieved by a steady flux of the surface, from a source at the front to the sink at the rear. Other swimmers change geometry cycling through a series of conformations of their shapes. Slender swimmers [7] of small volume-to-surface ratio use nonreciprocal bending beats to move $[3,4,8]$. Swimmers of moderate volume-tosurface ratio perform more complex shape changes to propel.

Technology [9] usually tries to mimic this variety [10] and rebuild [11,12] such swimmers using top-down approaches. Bibette et al. [12] built artificial flagella by connecting paramagnetic colloidal particles with DNA links to form a semiflexible chain attached to a larger particle at the rear of the swimmer. Bending waves induced via magnetic fields propel this biomimetic swimmer. Slender body hydrodynamics, i.e., treating the swimmer as a one-dimensional semiflexible object, could explain the propulsion mechanism. Its efficiency depends on the sperm number, i.e., the ratio of elastic bending torques to viscous torques of the fluid.

The use of bottom-up methods to build artificial swimmers is a rare exception successfully used only for high-Reynolds-number swimmers. Prominent examples of swimmers dynamically self-assembled from their components are magnetic snakes [13] and rings [14]. Here, we dynamically self-assemble an artificial low-Reynoldsnumber swimmer from the same paramagnetic particles used by Bibette. A complex magnetic field instead of DNA links forces the particles into two particle chains that bind side-by-side and form a ribbon. In contrast to Bibette's swimmer, our swimmer propels due to twist of the anisotopic cross section of the ribbon. A theoretical description beyond slender body hydrodynamics is needed to explain this ribbon-specific propulsion mechanism.

Mathematically, a ribbon differs from a curve since it additionally has a one-dimensional cross section. While a curve can bend and wind, a ribbon can additionally twist. The conformation of a ribbon can be described by its twist and its writhe. The sum of the twist (a local ribbon property) and the writhe (a global conformation property of the neutral line and thus a curve property) adds up to the link number. Beating cilia or flagella can be described by curves and propel by changes in writhe. In our ribbons, the neutral line remains a straight line and the writhe vanishes. The ribbon does not propel in the flat but in the twisted conformation. We can define the twist density as well as a flux of twist. Open ends of the ribbons allow a steady flux of twist through the ribbon. A parametrically modulated magnetic torque acting on the colloidal ribbon serves as a control parameter for the dynamically selfassembled shape. Shape transitions occur in the form of $\pi$ or $\pi / 2$ walls that travel along the ribbon. The number determining the propulsion is the ratio of twist and viscous torques, not the sperm number, and the assembly is propelled by a ribbon-specific mechanism.

The ribbon (Fig. 1) is formed in water from negatively charged $\left(\mathrm{COOH}^{-}\right)$paramagnetic Dynabeads $\mathrm{M}-270$ of radius $a=1.4 \mu \mathrm{m}$. The polystyrene beads have a core filled with superparamagnetic nanograins that render the 


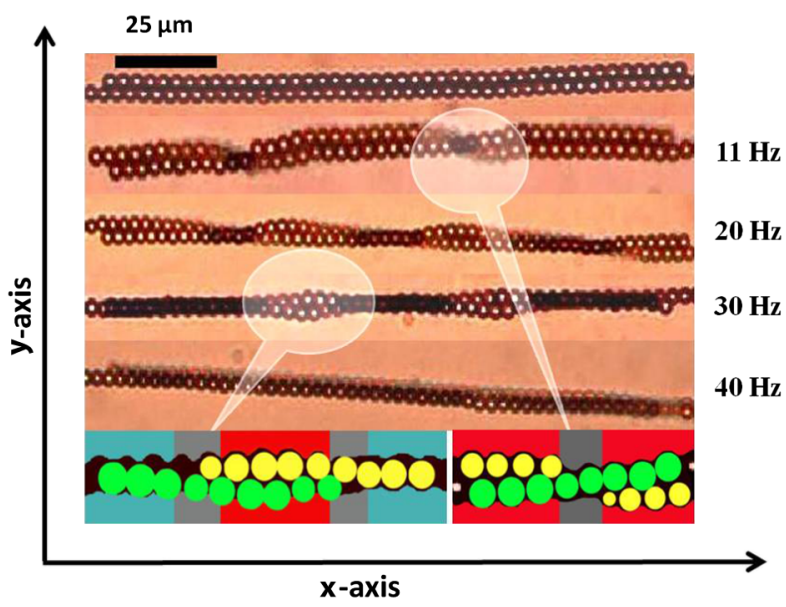

FIG. 1 (color online). Top: Microscopy image (top view) of a healed colloidal ribbon on a glass surface. The ribbon prepared at a frequency of $\Omega / 2 \pi<18 \mathrm{~Hz}$ and $\epsilon \approx-0.05$ lies in an untwisted conformation. $\Omega / 2 \pi=11-40 \mathrm{~Hz}$ : Microscopy images (top view) of the conformation of the ribbon for different frequencies and an eccentricity of $\epsilon \approx+0.05$. At the highest frequency $\Omega / 2 \pi=40 \mathrm{~Hz}$, the ribbon is standing on the glass surface. At low frequencies, lying domains (sketched in red in the scheme at the bottom) are separated by $\pi$ walls (sketched in gray) that, when approaching $\Omega / 2 \pi<40 \mathrm{~Hz}$, split into $\pi / 2$ walls separating lying from standing (cyan in the sketch) domains. Movies of the motion are shown in Ref. [16].

bead paramagnetic. The beads were diluted in Millipore water $\left(5 \times 10^{-6}\right.$ bead $\left./ \mathrm{ml}\right)$. Because of gravity along the $z$ direction, the colloids sedimented on top of a glass surface that was pretreated with a solution of polysodium 4-styrene sulfonate to prevent adhesion. Without a magnetic field, the lateral distribution of beads is random.

The magnetic field $\mathbf{H}(t)$ induces magnetic moments $\mathbf{m}(t)=\mu_{0} V \chi \mathbf{H}(t)$. Here, $\mu_{0}$ denotes the permeability of vacuum, $V$ the volume of the ensemble, and $\chi$ the effective susceptibility. The magnetic moments of the beads hence interact via dipolar interactions. The dimensionless Mason number $\mathcal{M}=\eta \Omega / \mu_{0} \chi^{2} H^{2}$ characterizes the ratio of viscous versus magnetic interactions, where $\eta=$ $10^{-3} \mathrm{~N} \mathrm{~s} \mathrm{~m}^{-2}$ denotes the water viscosity and $\Omega$ the modulation frequency at which the direction of the magnetic field changes. At the conditions used here, the Mason number is large $\mathcal{M}>1$ and the motion of the beads is with a lower rate $\omega<\Omega$ than that of the magnetic field because viscous forces are too strong to allow for a synchronous $(\omega=\Omega)$ motion. Frequencies $\Omega / 2 \pi>11 \mathrm{~Hz}$ were necessary to prevent disintegration of the ribbon.

Our system is driven by a magnetic field $\mathbf{H}(t)=\hat{H}\left[\cos \theta_{\mathrm{ext}} \mathbf{e}_{x}+\sin \theta_{\mathrm{ext}} \sqrt{2(1-\epsilon)} \mathbf{e}_{y} \cos (\Omega t)+\right.$ $\left.\sin \theta_{\text {ext }} \sqrt{2(1+\epsilon)} \mathbf{e}_{z} \sin (\Omega t)\right]$ of the average precession angle $\theta_{\text {ext }}$ and eccentricity $\epsilon$ that moves around the director along the $x$ axes. In this external field, we consider a pair of paramagnetic beads separated by the bond vector $\mathbf{r}_{b}$ enclosing a polar angle $\theta_{b}$ with the director and an
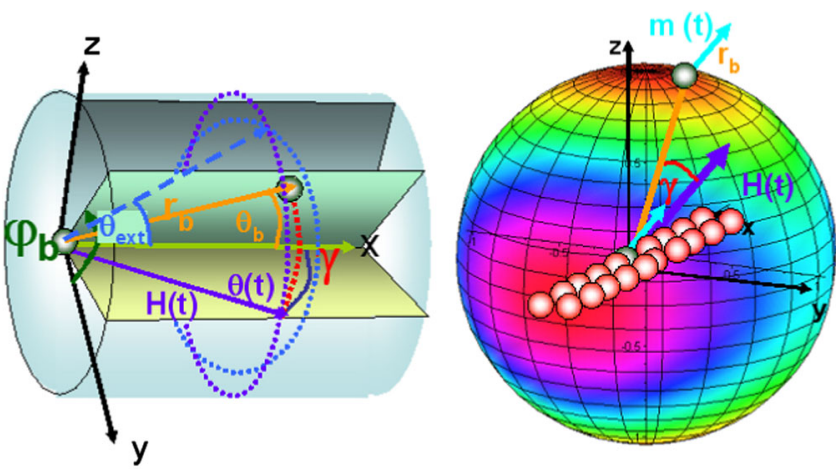

FIG. 2 (color online). The left scheme shows the definitions of angles defined between the director (green arrow), the magnetic field, and the bond vector. The magnetic field precesses on a dotted purple ellipse that deviates from a dotted blue circle but on average encloses the same angle $\theta_{\text {ext }}$ as the average blue field vector with the director. The right image shows the color coded averaged dipole interactions of a pair of dipoles in various directions for negative eccentricity. Dipoles form bonds in the attractive (purple) direction and avoid bonds in the repulsive (red) direction. Along the cyan direction, the averaged pair interaction is indifferent, and the formation of bonds or no bonds is decided by collective higher order effects.

azimuthal angle $\phi_{b}$ with the $y$ axis (Fig. 2). The dipolar energy of this pair is then given by $W(t)=$ $-\frac{\mu_{0} \chi_{\text {bead }}^{2} V^{2} H^{2}(t)}{4 \pi r_{b}^{3}} P_{2}(\cos \gamma(t))$, where $\gamma(t)$ denotes the angle between the magnetic field and the bond vector. The time averaging can be done by measuring both the orientation of the magnetic field and the bond vector with respect to the director. The time averaged dipolar interaction between two beads reads $\bar{W}=-\frac{\mu_{0} \chi_{\text {bead }}^{2} V^{2} \hat{H}^{2}}{4 \pi r^{3}}\left[P_{2}\left(\cos \theta_{\text {ext }}\right) P_{2}\left(\cos \theta_{b}\right)-\right.$ $\left.\epsilon P_{2}^{2}\left(\cos \theta_{\text {ext }}\right) P_{2}^{2}\left(\cos \theta_{b}\right) \cos \left(2 \phi_{b}\right)\right]$, where $P_{2}$ and $P_{2}^{2}$ are Legendre polynomials of degree 2 and associated Legendre polynomials of degree 2 and order 2, respectively. We assembled our ribbons with a magnetic field of $\hat{H}=2200 \mathrm{~A} / \mathrm{m}$, a precession angle of $\theta_{\text {ext }}=\pi / 6$, and negative eccentricities $\epsilon \approx-0.05$, for which the time averaged dipole interactions are attractive for a pair of beads separated along the $x$ direction $\left(\theta_{b}=0\right)$, weakly attractive to indifferent along the $y$ direction $\left(\theta_{b}=\pi / 2\right.$, $\left.\phi_{b}=0\right)$, and repulsive along the $z$ direction $\left(\theta_{b}=\pi / 2\right.$, $\left.\phi_{b}=\pi / 2\right)$. Since the interaction is weak along the $y$ direction, collective demagnetization effects involving three or more beads play a role along this direction. Yan et al. [15] used those collective effects to form hollow tubes. Here, collective effects lead to weak attraction in the $y$ direction between single chains but to repulsion between a ribbon and a third chain or an additional bead. We can create ribbons of typical length of up to 50 beads per chain. Defects in the form of vacancies on the ribbon or adsorbed beads starting a third chain can be eliminated by using an annealing procedure [16]. The top part in Fig. 1 shows an annealed ribbon. 
Once the ribbon is healed of defects, we switch to a positive eccentricity. This turns the $y$ direction repulsive and the $z$ direction attractive, favoring an upright orientation. Figure $1(40 \mathrm{~Hz}$ ) shows the conformation for $\epsilon \approx 0.05$ at $\Omega / 2 \pi=40 \mathrm{~Hz}$ and a magnetic field of $\hat{H}=2200 \mathrm{~A} / \mathrm{m}$ strong enough to force the ribbon against gravity into an upright conformation. The separation of the field modulation frequency from the rate of rotation is strong enough to wipe out all dynamic effects of the modulation onto the ribbon. This is no longer the case if we decrease the modulation frequency. Dynamic torques may now distort the conformation. The neutral line remains along the $x$ direction, and the conformation is entirely described by the angle $\phi_{b}(x)$ that the normal vector to the ribbon plane encloses with the $z$ direction. For frequencies below $\Omega / 2 \pi<40 \mathrm{~Hz}$, twist walls between subsequent quasistable orientations travel in the form of solitons with a speed $v_{\text {wall }}$ through the ribbon. These walls form via spontaneous symmetry breaking. The ribbon always rotates with the same sense as the magnetic field. Twist walls of left and right chirality nucleate with equal probability and travel into opposite directions on the ribbon. Once a steady state is reached, only walls of one chirality travel on one ribbon. For low frequencies $11 \mathrm{~Hz}<\Omega / 2 \pi<18 \mathrm{~Hz}$, such walls are $\pi$ walls connecting a lying ribbon section with another lying ribbon section. For frequencies $18 \mathrm{~Hz}<\Omega / 2 \pi<$ $28 \mathrm{~Hz}$, these $\pi$ walls split into two $\pi / 2$ walls, the first connecting a lying section with a standing section and the second connecting a standing section with a lying section twisted by $\pi$ with respect to the first lying section. For frequencies $28 \mathrm{~Hz}<\Omega / 2 \pi<40 \mathrm{~Hz}$, the $\pi / 2$ walls merge again to $\pi$ walls that connect two standing segments. Finally, above $\Omega / 2 \pi>40 \mathrm{~Hz}$, a standing flat ribbon remains.

In Fig. 3, we show spacetime plots of the angle $\phi_{b}(x, t)$ extracted from the videos for three frequencies. These plots show the behavior of the twist walls traveling at relatively large velocities and the forward propulsion with a much smaller propulsion velocity opposing the motion of the walls. A spatially more resolved version of the propulsion is shown in the inset of the top figure. The spacetime plots span the range $x_{b}(t)<x<x_{e}(t)$, where $x_{b, e}(t)=$ $x_{b, e}^{0}+v_{\text {prop }} t$ denote the propelling beginning and end of the ribbon. The color coding of the plot encodes the angle $\phi_{b}$, red colors correspond to a lying $\left(\phi_{b}=0\right)$ section, and cyan colors correspond to a standing $\left(\phi_{b}=\pi / 2\right)$ section. The dominating color shows whether the ribbon is lying or standing. Abrupt changes in color occur within the twist walls. $\pi$ walls connect regions of similar color, while the color changes from red to cyan when passing a $\pi / 2$ wall.

In Fig. 4, we plot the domain wall and the propulsion speed as a function of the modulation frequency. Domain walls and propulsion are observed in the frequency band $11 \mathrm{~Hz}<\Omega / 2 \pi<40 \mathrm{~Hz}$. We define a geometric propulsion efficiency $e=v_{\text {prop }} / v_{\text {wall }}$ analogous to that in
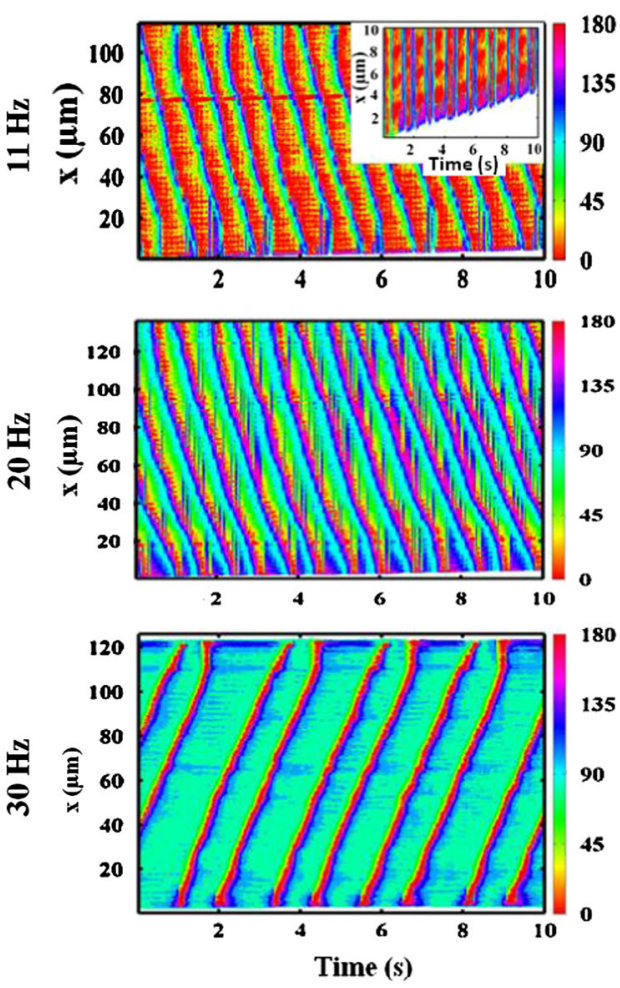

FIG. 3 (color online). Three spacetime plots of the orientation angle $\phi_{b}(x, t)$ for different frequencies $\Omega / 2 \pi$. The inset magnifies the region of one end of the ribbon.

Ref. [17] plotted in the third graph in Fig. 4 versus $\Omega / 2 \pi$. It measures the distance a ribbon propels during the motion of one domain wall by the wavelength. The efficiency increases with frequency. We express it in terms of the frequency $f$ of rotation of the ribbon and the density of walls $n=L / \lambda$ as $e=n v_{\text {prop }} / L f$, where $L$ is the length of the ribbon and $\lambda$ the wavelength. Since both the propulsion velocity and rotation frequency of the ribbon are fairly independent of the modulation frequency $\Omega$, the efficiency increases as the density of domain walls increases. It requires soft ribbons to achieve high densities of walls.

We can understand the behavior by a damped relaxation equation $\partial^{2} \phi_{b} / \partial t^{2}+\gamma \partial \phi_{b} / \partial t=-\delta F / \delta \phi_{b}(x)$, where $F=\int d x\left\{U_{\text {grav }}\left(\phi_{b}\right)+\Delta \chi U_{\text {magn }}\left(\phi_{b}, t\right)+\left(\partial \phi_{b} / \partial x\right)^{2} / 2\right\}$ is a rescaled functional of the field $\phi_{b}(x, t)$ with $U_{\text {grav }}\left(\phi_{b}\right)=\left|\sin \phi_{b}\right|$ the gravitational potential and $U_{\text {magn }}\left(\phi_{b}, t\right)=h_{+}^{2} \cos \left(2 \phi_{b}-2 \Omega t\right)+2 h_{+} h_{-} \cos \left(2 \phi_{b}\right)+$ $h_{-}^{2} \cos \left(2 \phi_{b}+2 \Omega t\right)$ the magnetic potential arising due to the magnetic torque density acting onto the anisotropic cross section of the two chains in a ribbon. $h_{+}$and $h_{-}$ are rescaled left and right circularly polarized magnetic field amplitudes. The prefactor $\Delta \chi$ denotes the anisotropy of the effective susceptibility of the ribbon cross section. The last term in the functional denotes the torsional rigidity. Following [18-20], we decompose the local orientation into a fast and a slow component $\phi_{b}=\phi_{s}+\phi_{f}$, expand 


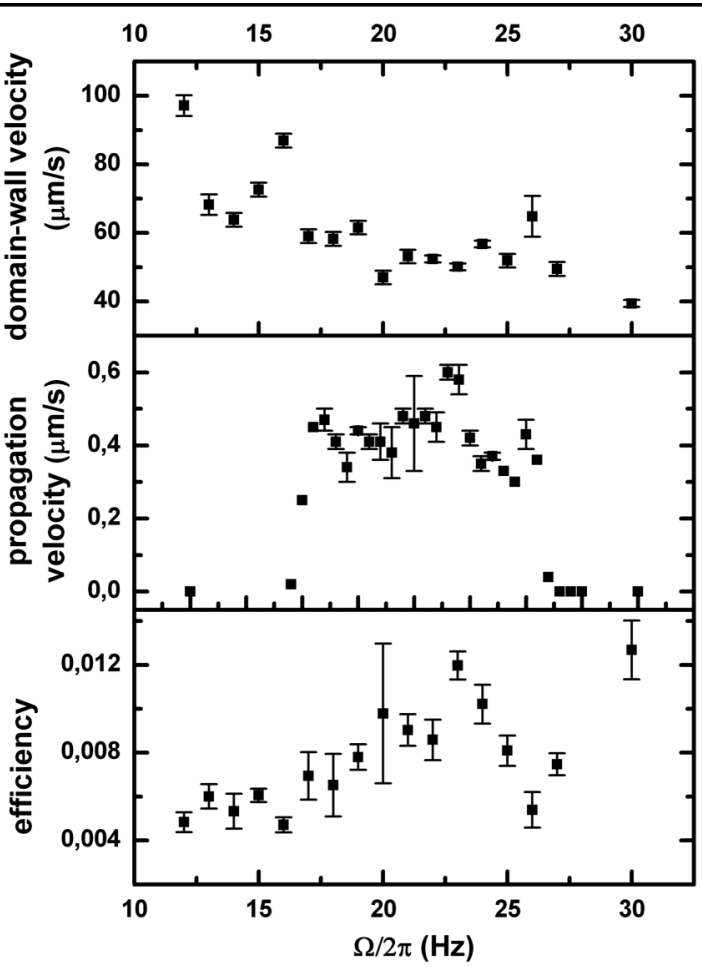

FIG. 4. Domain-wall velocity, propagation velocity, and the propulsion efficiency $e$ versus modulation frequency $\Omega / 2 \pi$. The error bars represent the standard deviation over 3-7 measurements for each point.

in terms of the fast component, and equate the resulting terms of the fast components and the time averaged slow components. This results in a time averaged equation of the slow component of the form $\partial^{2} \phi_{s} / \partial t^{2}+\gamma \partial \phi_{s} / \partial t-$ $\partial^{2} \phi_{s} / \partial x^{2}=f_{\text {eff }}-d U_{\text {eff }} / d \phi_{s}$ with an effective potential of the form $U_{\text {eff }}=\left|\sin \phi_{s}\right|+\Delta \chi 2 h_{+} h_{-} \cos \left(2 \phi_{s}\right)-$ $\Delta \chi^{2} h_{+}^{2} h_{-}^{2} \cos \left(4 \phi_{s}\right) / 2 \Omega^{2}$ and an effective force $f_{\text {eff }}=$ $\gamma \Delta \chi^{2}\left(h_{+}^{4}-h_{-}^{4}\right) / 2 \Omega^{3}$. The slow component tends to stay within the minima of the effective potential, while the fast component will algebraically vanish with increasing frequency, reducing fluctuations around the average orientation at large frequencies. Fluctuations render the time averaged cross section more isotropic. The anisotropy of the susceptibility also results self-consistently from depolarization fields of neighboring cross sections. When applying an elliptical external magnetic field with the major axis along the $z$ axis, then the eccentricity of the magnetic moment will be enhanced for an upright orientation and reduced for a horizontal orientation. This behavior can be modeled by a frequency dependent increasing $\Delta \chi(\Omega)$, which shifts the global minima from $\phi_{b}=0$ to $\phi_{b}=\pi / 2$ with frequency. Whether the effective potential exhibits minima at one or at both locations decides whether the domain walls are $\pi$ walls or $\pi / 2$ walls, respectively. For very high frequencies, the effective force and the fluctuations around the minima are too weak to overcome the barrier between two minima separated by $\pi$ and the ribbon is forced into the fully upright conformation, where no propulsion is possible.

In conclusion, dipolar interactions and gravity force an ensemble of paramagnetic beads into a ribbon. The ribbon changes from a flat lying ribbon via traveling twist walls toward a standing ribbon. For frequencies where domain walls are formed, the ribbon is propelled with an efficiency that scales with the domain-wall density.

This work was supported by the German Science Foundation via the priority program 1164; the SFB 840; FEDER-MINECO FIS2011-24540, MTM2009-12740C03-02, and PR2011-0123; FEDER-JA P09-FQM-4643; and by the Humboldt Foundation SPA 1146358 STP.

*Thomas.Fischer@uni-bayreuth.de

[1] H. C. Berg, Nature (London) 249, 77 (1974).

[2] C. Eloy and E. Lauga, Phys. Rev. Lett. 109, 038101 (2012).

[3] J. Lighthill, SIAM Rev. 18, 161 (1976).

[4] J. Lighthill, J. Eng. Math. 30, 35 (1996).

[5] O. Pironneau and D.F. Katz, J. Fluid Mech. 66, 391 (1974).

[6] A. T. Chwang and T.Z.T. Wu, J. Fluid Mech. 67, 787 (1975).

[7] H. Wada and R. R. Netz, Phys. Rev. Lett. 99, 108102 (2007).

[8] J. J. L. Hidgon, J. Fluid Mech. 94, 331 (1979).

[9] L. Zhang, J. J. Abbott, L. Dong, B. E. Kratochvil, D. Bell, and B.J. Nelson, Appl. Phys. Lett. 94, 064107 (2009).

[10] A. Beveratos, I. Sagnes, S. Haliyo, S. Régnier, G. Hwang, R. Braive, L. Couraud, A. Cavanna, O. Abdelkarim, and I. Robert-Philip, International Journal of Robotics Research 30, 806 (2011).

[11] R. Livanovics and A. Cebers, Phys. Rev. E 85, 041502 (2012).

[12] R. Dreyfus, J. Baudry, M. L. Roper, M. Fermigier, H. A. Stone, and J. Bibette, Nature (London) 437, 862 (2005).

[13] A. Snezhko, I. S. Aranson, and W. K. Kwok, Phys. Rev. Lett. 96, 078701 (2006).

[14] B. A. Grzybowski, J.A. Wiles, and G. M. Whitesides, Phys. Rev. Lett. 90, 083903 (2003).

[15] J. Yan, M. Bloom, S. C. Bae, E. Luijten, and S. Granick, Nature (London) 491, 578 (2012).

[16] See Supplemental Material at http://link.aps.org/ supplemental/10.1103/PhysRevLett.110.168302 for description of the ribbon assembly and videos of the ribbon.

[17] D. Tam and A. E. Hosoi, Phys. Rev. Lett. 98, 068105 (2007).

[18] P. L. Kapitza, Sov. Phys. JETP 21, 588 (1951).

[19] P. L. Kapitza, Usp. Fiz. Nauk 44, 7 (1951).

[20] Yu. S. Kivshar, A. Sanchez, and L. Vazquez, Phys. Rev. A 45, 1207 (1992). 